\title{
核聚变堆用铇表面超精密抛光的研究现状与趋势“
}

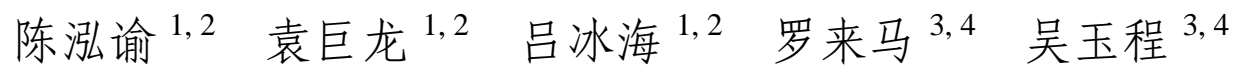 \\ (1. 浙江工业大学机械工程学院 杭州 310023; \\ 2. 浙江工业大学超精密加工研究中心 杭州 310023; \\ 3. 合肥工业大学材料科学与工程学院 合肥 230009 ;
}

4. 合肥工业大学有色金属与加工技术国家地方联合工程研究中心 合肥 230009)

\begin{abstract}
摘要: 铇作为未来核聚变堆中最有前景的面向等离子体材料, 在反应堆工况下将承受高能粒子的辐照冲击。表面质量的好坏会直 接影响材料的氢/氦滞留行为和辐照损伤程度, 进而影响聚变堆的安全性和可靠性。现阶段, 针对铇的抗辐照改性研究主要着眼于 材料的成分、结构和组织设计, 关于机械加工对材料表面抗辐照改性的研究甚少。文章聚焦前沿科学问题, 从机械加工角度分析 核材料领域科学问题, 结合国内外相关研究成果及核聚变堆用铇(PFM-W)的机械加工现状, 阐述了 PFM-W 表面超精密抛光的必 要性。通过对比不同抛光方法, 提出了磁流变抛光和力流变抛光是较为适合 PFM-W 表面超精密加工的观点, 并对未来 PFM-W 表面超精密抛光研究趋势进行了分析, 重点在抛光方法的探索以及抛光后材料表面质量对抗辐照性能影响的研究。
\end{abstract}

关键词: 铇; 超精密抛光; 表面质量; 抗辐照性能

中图分类号: TH161

\section{Research Status and Trend of Ultra-precision Polishing on Tungsten Surface for Fusion Reactors}

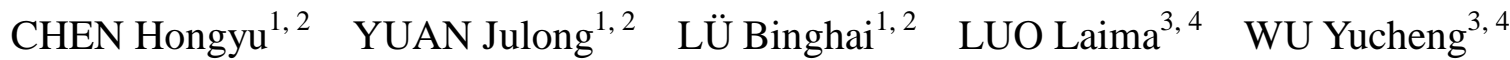

(1. College of Mechanical Engineering, Zhejiang University of Technology, Hangzhou 310023;

2. Ultra-Precision Machining Center, Zhejiang University of Technology, Hangzhou 310023;

3. School of Materials Science and Engineering, Hefei University of Technology, Hefei 230009;

4. National-Local Joint Engineering Research Center of Nonferrous Metals and Processing Technology, Hefei

University of Technology, Hefei 230009)

\begin{abstract}
Tungsten, as the most promising plasma facing material in the future fusion reactor, will withstand the irradiation impact of high energy particles under reactor condition. The surface quality of material will directly affect the hydrogen/helium retention behavior and the degree of radiation damage, thus affecting the safety and reliability of fusion reactor. At the present stage, the research on irradiation resistance modification of tungsten mainly focuses on the composition, structure and organization design of the material, few studies have been proceeded on the irradiation resistance modification of material surface. Focusing on the frontier scientific problems, the study intends to analyze the scientific problems in the field of nuclear materials from the perspective of mechanical processing, describes the necessity of ultra-precision polishing on the tungsten surface for nuclear fusion reactor based on the domestic and international research results and machining status of tungsten for nuclear fusion (PFM-W). Through comparing with different polishing methods, the viewpoint that magneto-rheological finishing and force-induced rheological polishing are more suitable for ultra-precision polishing of PFM-W surface. In addition, the future research trend of ultra-precision polishing of PFM-W surface is analyzed, with emphasis on the exploration of the polishing method and the influence of the surface quality of the polished material on the radiation resistance.
\end{abstract}

Key words: tungsten; ultra-precision polishing; surface quality; radiation resistance

* 国家自然科学基金(51905485)和浙江省自然科学基金(LR17E050002)资 助项目。20200302 收到初稿, 20200608 收到修改稿 


\section{0 前言}

核聚变能由于其经济、清洁、高的能量密度以 及可持续发展等优点, 被认为是可以最终解决人类 能源问题的主要途径之一。自 20 世纪 50 年代初, 前苏联科学家提出托卡马克(Tokamak)这一概念至 今, 科学家们进行了长期的探索研究, 旨在全面验 证聚变能和平利用的科学可行性和工程可行性。我 国于 21 世纪初先后建成了环流器二号(HL-2M)和东 方超环(EAST)核聚变试验装置, 并于 2006 年正式 加入了国际热核试验堆计划(ITER), 多年来成果显 著。2017 年, 各国专家发表了《北京聚变宣言》, 支持中国建设 “中国聚变工程试验堆(CFETR)”, 为 ITER 与未来核聚变电站之间建起桥梁, 图 1 为我国 核聚变发展战略规划图 ${ }^{[2]}$ 。

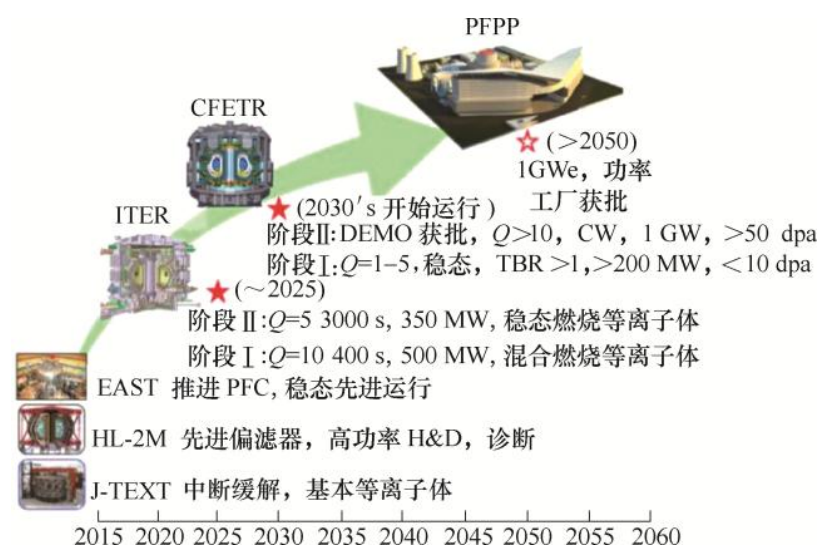

图 1 我国核聚变发展战略规划图 ${ }^{[2]}$

在忥-気(D-T)聚变反应过程中, 面向等离子体 材料(Plasma facing materials, PFMs)需要承受高热 负荷 $\left(10 \sim 20 \mathrm{MW} \cdot \mathrm{m}^{-2}\right)$ 、高氢/氦等离子体通量 $\left(1 \times 10^{20} \sim 1 \times 10^{24} \mathrm{~m}^{-2} \cdot \mathrm{s}^{-1}\right)$ 和高能量 $(14.1 \mathrm{MeV})$ 中子辐 照的考验 ${ }^{[3-5]}$ 。这就需要材料具有熔点高、热导率好、 抗热冲击性能强等性能 ${ }^{[6-8]}$ 。铇(W) 由于其高熔点 $\left(3410{ }^{\circ} \mathrm{C}\right)$ 、高热导率、低的热膨胀系数、优异的抗 溅射性能以及気滞留低且不与 $\mathrm{H}$ 反应等优点, 被认 为是未来核聚变反应堆中最有前景的 $\mathrm{PFM}^{[9-10]}$ 。ITER 装置中, 偏滤器的内外垂直靶、圆顶伞、粒子反射片

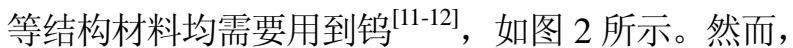
铇的氢/氦滞留以及辐照损伤一直是需要克服的难点。 反应堆工况下，铇表面会直接暴露在氢、氦等高能粒 子辐照环境中, 导致起泡、肿胀、硬化、脆化等辐照 损伤的产生，更严重时甚至在表面形成纳米丝状结 构, 改变材料表面物理和化学性质如热导率、机械性 能等, 缩短部件使用寿命, 影响热核聚变堆的安全性
和可靠性 ${ }^{[13-14]}$ 。图 $3 \mathrm{a}$ 和 $3 \mathrm{~b}$ 分别为铇表面受到氢 $/$ 氦辐 照损伤后产生的氢泡和纳米丝状结构图 ${ }^{[15-16]}$ 。因此, 研究核聚变堆用铇的辐照损伤及氢/氦滞留行为, 提升 其抗辐照性能便显得为尤其重要。

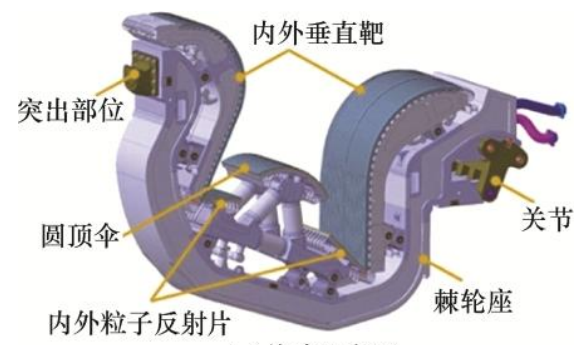

(a) 偏滤器主图

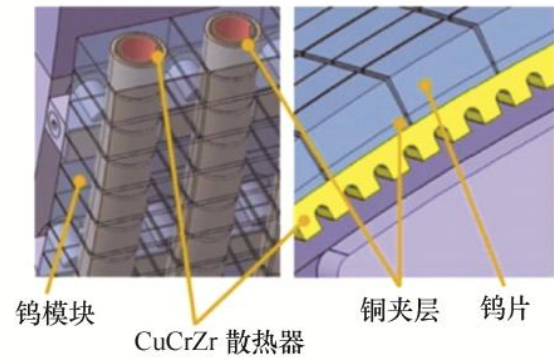

(b) 内外垂直靶几何结构图 (c) 圆顶伞和粒子反射片结构图

图 2 ITER 偏滤器及其主要部件结构示意图 ${ }^{[12]}$

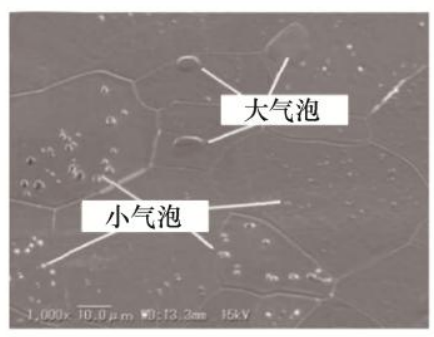

(a) 氢辐照后表面的气泡

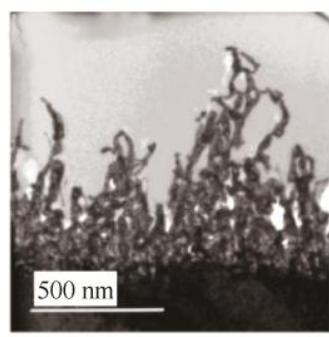

(b) 氦辐照后产生纳米丝状结构
图 3 铇的氢/氦辐照损伤 ${ }^{[15-16]}$

\section{1 核聚变堆用铇表面抛光必要性}

PFM-W 表面质量的好坏会直接影响材料的氢/ 氦滞留行为 ${ }^{[17-18]}$ ，表面粗糙的工件在实际工况下会 捕获更多的氢/氦粒子, 加剧辐照损伤程度。目前国 内外学者主要还是从材料学角度研究铇的抗辐照行 为, 通过成分、结构和组织设计对铇及其合金进行 改性研究以期提高其抗辐照能力, 关于机械加工对 PFM-W 表面抗辐照改性的研究甚少。

\section{1 表面质量对材料抗辐照行为的影响}

氢/氦辐照下, 材料中点阵原子会引起一系列的 级联碰撞并产生离位损伤, 导致大量辐照缺陷的产 生。PFM-W 的氢/氦滞留行为与辐照缺陷以及材料 的本征缺陷有关。 
SUGANO 等 ${ }^{[19]}$ 和 CAO 等 ${ }^{[18]}$ 分别对 bcc 结构的 铁和铁铬合金进行了氦辐照研究, 一致认为辐照后 材料中氦的滞留量与其表面质量有关。SUGANO 等通过试验研究发现 Fe 的 TDS(热脱附)曲线图中 存在五种峰, 依次与材料表面、 $\mathrm{He} n \mathrm{~V}(2 \leqslant n \leqslant 6)$ 、 $\operatorname{HenV} m(1 \leqslant n, 2 \leqslant m) 、 \gamma$ 相变和氦泡有关, 如图 4 所示, 其中 $\mathrm{HeV}$ 为氦与空位相结合形成的复合体。 $\mathrm{Fe}-\mathrm{Cr}$ 合金的 TDS 曲线图与 $\mathrm{Fe}$ 类似。研究表明, $300 \sim 650 \mathrm{~K}$ 温度下合金加热后脱附的氦(即氦辐照 过程中滞留的氦)主要与材料表面有关。XU 等通 过试验和理论分析得出了类似结论, 认为在低于 $800 \mathrm{~K}$ 下检测到的热脱附氦曲线与材料表面质量 有关, 如图 5 所示, 其中 $\mathrm{He}^{+}$离子能量 $0.15 \mathrm{keV}$, 辐照剂量 $5.0 \times 10^{19} \mathrm{He}^{+} / \mathrm{m}^{2}$ 。

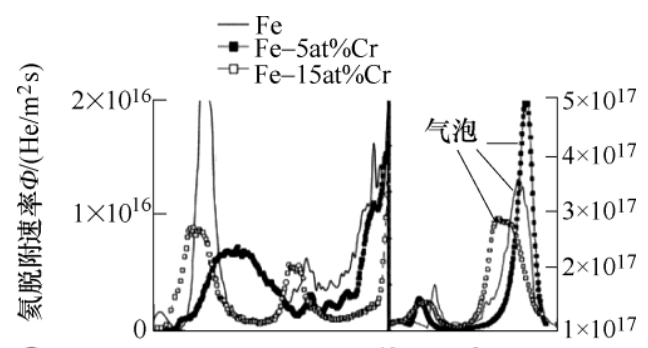

(a) 辐照剂量 $10^{19} \mathrm{He}^{+} / \mathrm{m}^{2}$

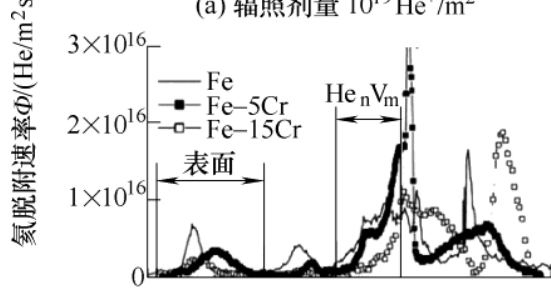

(b) 辐照剂量 $10^{18} \mathrm{He}^{+} / \mathrm{m}^{2}$

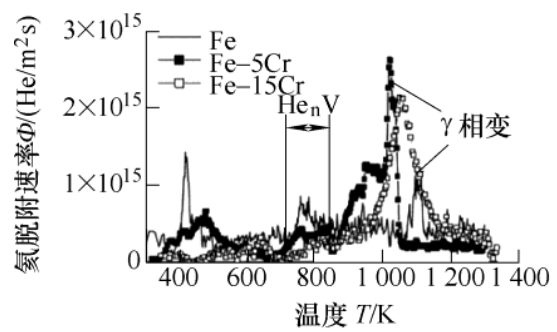

(c) 辐照剂量 $10^{17} \mathrm{He}^{+} / \mathrm{m}^{2}$

图 4 室温下 $8 \mathrm{keV}$ 氦辐照后, $\mathrm{Fe} 、 \mathrm{Fe}-5 \mathrm{Cr}$ 和 $\mathrm{Fe}-15 \mathrm{Cr}$ 的热脱附曲线图 ${ }^{[19]}$

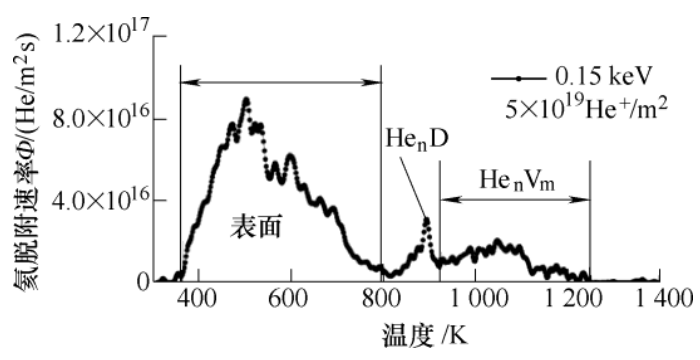

图 5 氦辐照后铁的热脱附曲线图 ${ }^{[18]}$

本课题组此前对钨合金进行了氛滞留(氢辐照)
试验, 相关工作在日本京都大学原子炉实验所进行。 结果表明，不同辐照剂量、不同成分的铇合金在 298 600 K 温度区间均会产生一个明显的低温脱 附峰，且大部分氢同位素的滞留与该区间有关。当 辐照剂量由 $1.0 \times 10^{20}$ 增至 $1.0 \times 10^{21} \mathrm{D}_{2}{ }^{+} / \mathrm{m}^{2}$ 时, 铇及 其合金的脱附峰均有一定程度地拉长, 如图 6 所示。 随着辐照剂量的增加, 辐照损伤引入的缺陷密度和 尺寸增大，新的位错环和空位的产生会进一步地吸 收気, 增大其滞留量。気滞留试验是一个先注気随 后热脱附的过程。热脱附 TDS 曲线低温区间的脱附 峰主要与材料表面质量(表面形貌、表面粗糙度、本 征缺陷)有关, 而高温区间的脱附峰则主要与辐照引 入的缺陷有关。通过机械加工方式改善材料表面形 貌, 降低表面粗粘度, 在一定程度上能够减少材料 对氛原子的捕获, 进而提高材料的抗辐照性能。

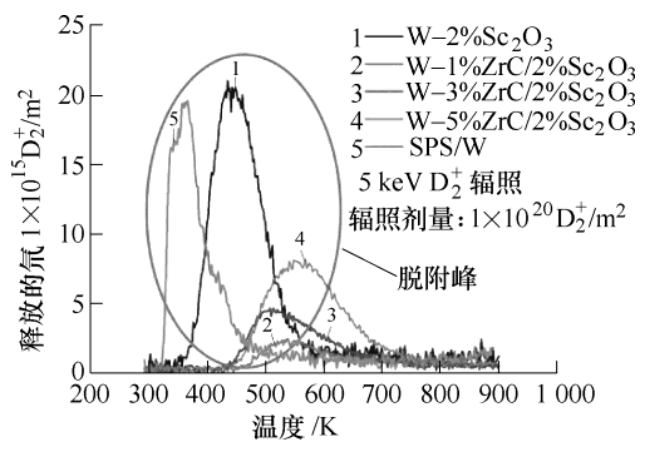

(a) 辐照剂量 $1 \times 10^{20} \mathrm{D}_{2}^{+} / \mathrm{m}^{2}$

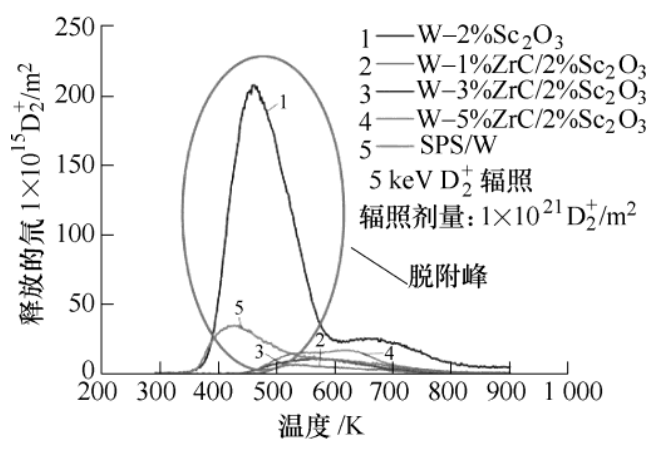

(b) 辐照剂量 $1 \times 10^{21} \mathrm{D}_{2}^{+} / \mathrm{m}^{2}$

图 6 铇及其合金的热脱附 TDS 曲线图

PFM-W 为轧制后的 bcc 材料。因此, 参照同种晶 格类型的铁在 $400 \sim 800 \mathrm{~K}$ 之间的氦滞留行为并结合 本课题组之前的研究结果 ${ }^{[20]}$, 有必要对 PFM-W 的表 面质量与抗氢(氦辐照性能之间的关系进行重点研究。

\subsection{PFM-W 精密加工现状}

现阶段, 试验人员在进行钨辐照试验之前通常 采用手工抛光对试样进行简单处理, 对材料表面粗 鋉度和形貌并无具体要求或统一标准。而手工操作 的加工效率低, 对操作人员的技术与经验依赖性大, 且难以保证抛光质量与重复性。辐照试验时, 同组 试样的材料表面质量一致性不佳也会导致试验结果 


\section{存在一定的偏差。}

目前国内外关于 PFM 的机械加工研究较少, 中 国科学院等离子体物理研究所(ASIPP)服役的 EAST 装置中的 PFM-W 主要以磨削加工为主, 实际工件 由安泰科技股份有限公司提供, 工件表面加工参数 表面粗楉度 $\left(R_{a}\right)$ 为 $1.6 \mu \mathrm{m}$ 。课题组对安泰提供的模 拟试验用铇铜穿管小模块需要承受辐照考验表面的 粗粘度进行了检测, 发现磨削后的钧表面有明显的 磨削轨迹存在, 最佳 $R_{a}$ 仅为 $606.7 \mathrm{~nm}$ 左右, 如图 7 所示。可见, 现阶段核聚变研究领域对 PFM-W 机 械加工后表面粗䊁度和表面形貌的问题并没有引起 重视。关于机械加工对 PFM-W 表面质量及抗辐照 性能改善的相关研究亦鲜见报道。因此, 结合表面 质量对材料抗辐照行为的影响以及目前 PFM-W 机 械加工现状, 通过超精密抛光改善材料表面形貌, 优化表面质量从而提高材料抗氢/氦辐照性能(抗辐 照改性)是具备理论基础和可行性的。

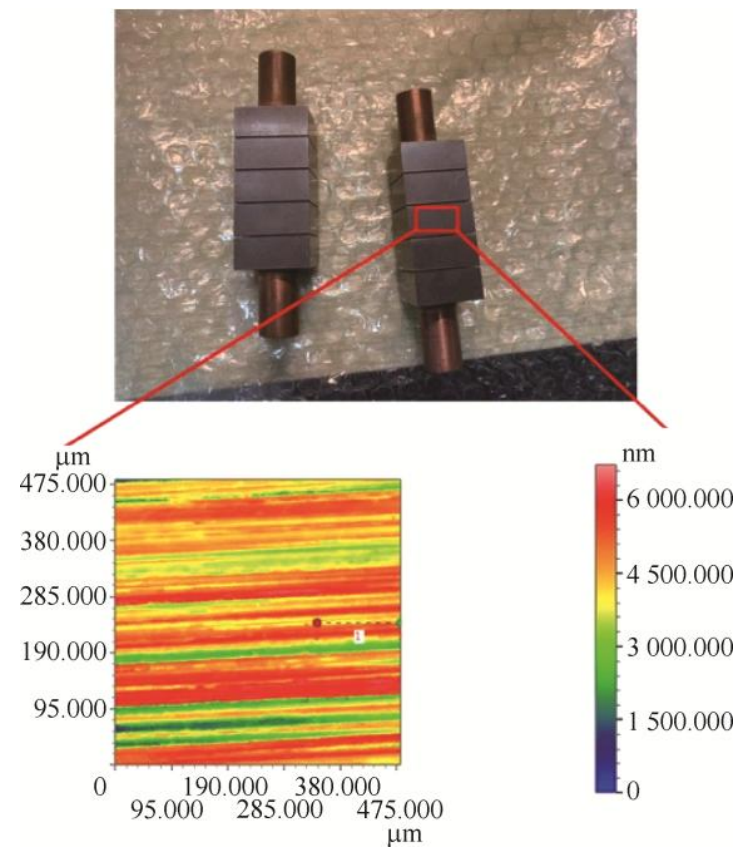

图 7 铇铜穿管小模块及其表面粗䊅度图

\section{2 国内外抛光工艺研究现状}

铇由于其硬脆特性存在机械加工难度大的问 题, 稍有不慎就会引起表面的磨损或破坏。目前, 从高效高精度考虑, 国内外针对钧的抛光主要采用 化学机械抛光和电化学抛光, 而有关磁流变抛光技 术、力流变抛光、离子束抛光、激光技术抛光等新 型加工方法应用研究则鲜有报道。

\section{1 化学机械抛光}

化学机械抛光(Chemical mechanical polishing,
CMP)是将磨粒的机械研磨作用与氧化剂的化学作用 相结合, 从而达到高去除速率和良好平面性效果的抛 光技术 ${ }^{[21]}$ 。由于氧化铇比钧更柔软且更易去除, 相应 的机械磨除作用也更显著。在实际抛光中, 如何快速 有效地形成表面氧化层是实现高去除率和高抛光性 能的关键。因此, 选择合适的氧化剂至关重要。

化学机械抛光液的不同成分将会直接影响到铇 的抛光效果。梅广益等 ${ }^{[22]}$ 通过调配不同的氧化剂 $\left(\mathrm{H}_{2} \mathrm{O}_{2}\right)$ 、蚀刻剂 $\left(\mathrm{Fe}\left(\mathrm{NO}_{3}\right)_{3}\right)$ 和配位剂(乳酸、 $\mathrm{SSA}$ )组 成特定抛光液对铇进行了抛光试验研究, 主要化学 反应方程式为

$$
\mathrm{W}+\mathrm{H}_{2} \mathrm{O}_{2} \rightarrow \mathrm{W}_{x} \mathrm{O}_{y}+\mathrm{H}_{2} \mathrm{O}
$$

当 $\mathrm{H}_{2} \mathrm{O}_{2}$ 和 $\mathrm{Fe}\left(\mathrm{NO}_{3}\right)_{3}$ 含量较低时, 随着两者含量的 增加, 抛光效果近似线性的提高。而当含量达到一 定值后, 随着 $\mathrm{H}_{2} \mathrm{O}_{2}$ 的继续增加, 抛光效果反而下降。 结果表明, 当 $\mathrm{H}_{2} \mathrm{O}_{2}$ 含量为 $4 \%, \mathrm{Fe}\left(\mathrm{NO}_{3}\right)_{3}$ 含量为 $0.05 \%$ 时抛光效果最佳, 表面粗䊁度 $R_{\mathrm{a}}$ 能够达到 $0.198 \mathrm{~nm}$ 。贾英茜等 ${ }^{[23]}$ 采用碱性抛光液对铇进行了 抛光, 主要组分为氧化剂 $\left(\mathrm{H}_{2} \mathrm{O}_{2}\right)$ 、纳米 $\mathrm{SiO}_{2}$ 水溶胶 磨料及有机碱。试验表明, 在抛光液配制过程中还 需考虑组分之间的相互作用。SEO 等 ${ }^{[24]}$ 采用 $\mathrm{H}_{2} \mathrm{O}_{2}$ 和 $\mathrm{Fe}\left(\mathrm{NO}_{3}\right)_{3}$ 混合氧化剂对铇进行了化学机械抛光和 电化学特性研究。结果表明, $5 \% \mathrm{Fe}\left(\mathrm{NO}_{3}\right)_{3}+5 \% \mathrm{H}_{2} \mathrm{O}_{2}$ 的混合氧化剂具有较好的去除率。LIM 等 ${ }^{[25]}$ 研究发 现, 抛光液的化学成分对铇表层氧化态 $\left(\mathrm{W} 、 \mathrm{WO}_{2}\right.$ 、 $\left.\mathrm{WO}_{3}\right)$ 和微观结构有很大的影响。 $\mathrm{Fe}\left(\mathrm{NO}_{3}\right)_{3}$ 会在铇表 面形成较致密的氧化层, 而 $\mathrm{H}_{2} \mathrm{O}_{2}$ 则会在铇表面形成 多孔氧化层, 如图 8 所示。抛光过程中, 快速有效 地形成表面氧化层是获得高去除率的关键因素，表 面氧化层的化学成分和微观结构也影响铇的去除 率。因此, 从氧化动力学的角度考虑, 当抛光液中 添加混合氧化剂时抛光的去除率更好。

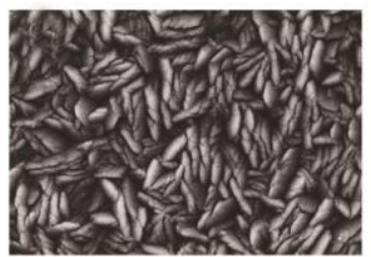

(a) 原始铇片

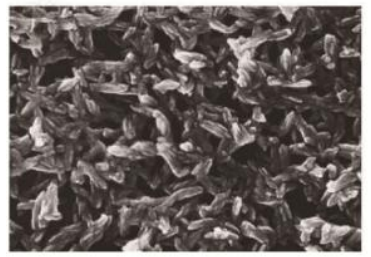

(c) $\mathrm{H}_{2} \mathrm{O}_{2}$

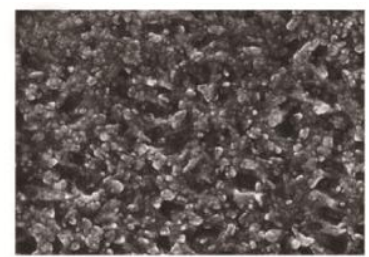

(b) $\mathrm{Fe}\left(\mathrm{NO}_{3}\right)_{3}$

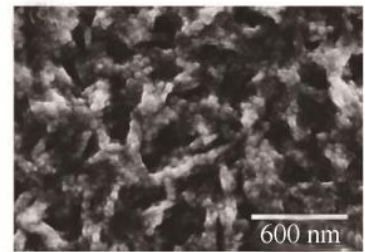

(d) $\mathrm{Fe}\left(\mathrm{NO}_{3}\right)_{3}$ 和 $\mathrm{H}_{2} \mathrm{O}_{2}$
图 8 铇在不同氧化剂溶液中氧化后的 SEM 图 ${ }^{[25]}$ 
$\mathrm{Fe}\left(\mathrm{NO}_{3}\right)_{3}$ 和 $\mathrm{H}_{2} \mathrm{O}_{2}$ 是抛光液能够实现铇表面高 效化学机械抛光的主要成分, 但 $\mathrm{H}_{2} \mathrm{O}_{2}$ 的蒸气压比水 高, 在 $60{ }^{\circ} \mathrm{C}$ 以上容易分解为氧气, 在催化剂 $\mathrm{Fe}\left(\mathrm{NO}_{3}\right)_{3}$ 的作用下, 这种分解速率会进一步增强, 导致氧气泡的形成。JEONG 等 ${ }^{[26]}$ 采用 $\mathrm{Fe}\left(\mathrm{NO}_{3}\right)_{3}$ 和 $\mathrm{H}_{2} \mathrm{O}_{2}$ 对铇进行了化学机械抛光, 发现抛光液存在磨 粒颗粒团聚的现象, 该现象与氧气泡的形成有关。氧 气泡能够增加磨粒的亲水性, 增强磨粒和原位生成氧 泡之间的粘附力, 进而导致抛光液中磨粒的团聚。

LARSEN-BASSE 等 ${ }^{[27]}$ 对铇化学机械抛光过程 中可能出现的磨损现象进行了研究, 认为 CMP 是 一个磨料去除针化膜和裸表面与抛光液主动-被动 反应重新形成钝化膜的协同过程, 两种反应都发生 在毫秒或更短的时间尺度内, 其去除率比分步加工 高出几个数量级。因此, 可以从针化条件、材料纯 度、磨粒浓度、抛光速度以及表面相互作用角度考 虑优化工艺。

化学机械抛光综合了化学能和机械能各自的优 势, 具有在较低成本下获得较低表面粗粘度和较高 材料去除率以及实现全局平坦化等优点。但也存在 不足之处, 如使用后的碱性抛光液腐蚀性强且难以 处理, 造成一定的环境污染。

\section{2 电化学抛光}

电化学抛光(Electrochemical polishing, ECP), 又称 电解抛光, 是指将金属在特定组分的溶液中进行特殊 阳极处理, 从而获得平滑、光亮表面的抛光工艺。该 过程不存在机械力的作用, 因而不会产生金属的表面 变形或加工硬化层, 也不带入残余应力, 具有效率高、 操作简便、不受材料制约等优点。其原理目前被业界 广泛公认的主要为黏膜理论 ${ }^{[28]}$ 和钝化膜理论 ${ }^{[29]}$ 。

DENG 等 ${ }^{[30]}$ 针对铇的电解拋光效率和表面质量 进行了相关研究, 就电流和电势提出了一种高效抛 光工艺: 首先采用电流主导 $(500 \mathrm{~mA})$ 进行 $3 \mathrm{~min}$ 电 解抛光, 目的是迅速去除表面磨痕和亚表面损伤; 随后采用电势主导 $(60 \mathrm{~V})$ 进行 $20 \mathrm{~min}$ 的电解抛光, 进一步降低表面粗糙度以得到超光滑的材料表面, 最终的 $R_{a}$ 为 $17.6 \mathrm{~nm}$ 。该团队的 WANG 等 ${ }^{[31]}$ 在上 述研究基础上通过 $10 \mathrm{~min}$ 的电解抛光, 在 $60 \mathrm{~V}$ 电 势作用下得到了表面粗䊁度 $S a$ 仅为 $3.73 \mathrm{~nm}$ 的光滑 镜面, 证明了电解抛光的高效性, 但材料表面仍存 在致密的小坑, 如图 9 所示。除此之外, WANG 等 还对不同电势下铇电解加工过程中的阳极行为进行 了探索, 研究分析了不同电势下材料表面形貌、粗 䊁度和电流的变化, 并根据抛光后铇表面形貌的差 异将不同电势下铇的电解抛光过程分为蚀刻、光亮
和点蚀 3 个阶段。结果表明, 如若需要得到光滑无 麻点的铇表面, 需要将电势控制在 5 25 V 的范围 内, 以便平衡腐蚀和抛光的过程。

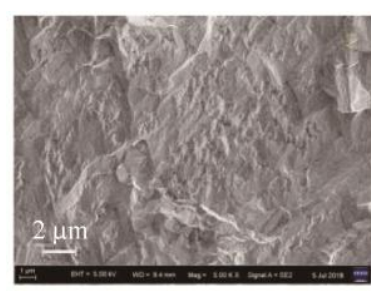

(a) 原始铇表面

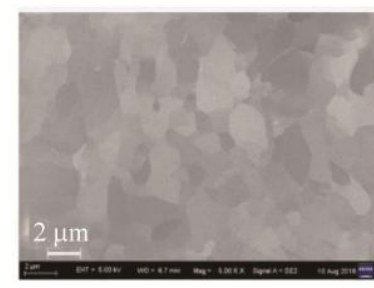

(c) 电势 $10 \mathrm{~V}$

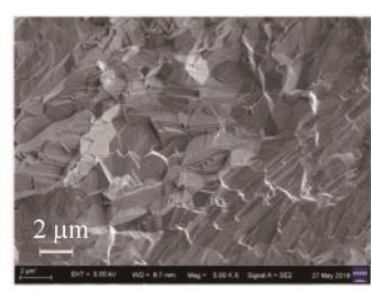

(b) 电势 $3 \mathrm{~V}$

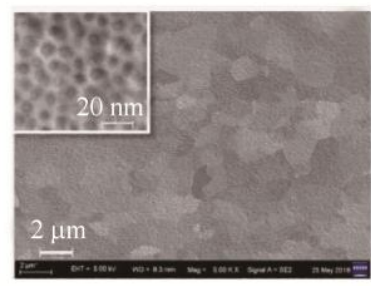

(d) 电势 $60 \mathrm{~V}$
图 9 铇电化学抛光后的表面形貌 SEM 图 ${ }^{[31]}$

现阶段国内外对于铇的电解抛光仍以碱性电解 抛光液为主, 而酸性体系应用于铇电解抛光的相关 报道较少。刘奉妍等 ${ }^{[32]}$ 采用硫酸-甲醇-柠檬酸钠电 解液体系对铇进行了电解抛光, 研究了柠檬酸钠浓 度对试样表面微观形貌和表面粗糙度的影响和作用 机理。结果表明, 在电解液中柠檬酸钠浓度为 $0.25 \mathrm{~mol} / \mathrm{L}$ 时试样可获得最佳表面粗粘度和较好表 面形貌， $R_{a}$ 仅为 $10 \mathrm{~nm}$ 。王雪等 ${ }^{[33]}$ 对 $\mathrm{NiW}$ 合金基带 的电化学抛光过程进行了研究, 发现适当浓度的抛 光液可在阳极表面生成致密且具有黏性的铇酸胶体 层, 对材料表面光亮化起到重要作用, 有助于获得 较好的抛光效果。而当抛光液浓度较低时材料表面 会形成过量的氧化物和 $\mathrm{H}_{2} \mathrm{WO}_{4}$, 造成表面选择性溶 解过程紊乱, 不利于抛光的进行。

电化学抛光因其成本低、效率高、劳动强度小、 不受抛光工件形状限制及抛光质量好等优点得到了 制造业的广泛认可。随着电化学抛光技术的不断发展 和完善, 相继出现了与磁场、脉冲、超声波等技术相 结合的复合电化学抛光新技术 ${ }^{[34]}$ 。然而, 该工艺仍旧 存在缺点, 包括加工工件的表面质量与材料本身的组 织均匀性和纯度相关, 工件尺寸和几何形状的精确度 较难控制以及抛光液的环境污染等问题。

化学机械抛光和电化学抛光的抛光液势必会腐 蚀铇, 导致材料表面氧化铇的形成, 相当于改变了 材料的表层材质(化学产物污染), 这在 PFM-W 的加 工中是不可容忍的 ${ }^{[35-36]}$ 。

\section{3 磁流变抛光}

磁流变抛光技术(Magneto-rheological finishing, 
MRF) 是 20 世纪 90 年代由 KORDONSKI 及其合作 者基于磁流变效应提出的一种新型柔性抛光方法。 加工过程中, 法向压力极低, 磨粒压入工件的深度 较浅, 主要通过剪切作用实现材料去除, 从而获得 高质量表面，且几乎不产生表面和亚表面损伤。同 时, 由于磁场约束, 磨粒稳定分布于工作界面, 能 够有效提高加工效率和精度 ${ }^{[37-38]}$ 。

磁流变抛光技术常用于光学器件的加工, 目前 暂无加工钨方面的研究报道。由于磁场效应, 磁流 变抛光液呈现出 Bingham 流体特性, 发生流变的抛 光液流经抛光区域时, 会在抛光盘上形成固态核, 使得磁流变抛光效率大大提高。彭小强等 ${ }^{[39]}$ 对磁流 变抛光液在抛光区域的固态核分布进行了理论分 析, 依据 Preston 方程从工件表面所受正压力出发, 建立了磁流变抛光的材料去除数学模型, 结合模型 与试验, 推导出在工艺条件下准 Preston 系数的平均 值为 $K=1.35 \times 10^{-11} \mathrm{~m}^{2} \cdot \mathrm{N}^{-1}$, 为磁流变数控化提供了 理论依据。KHATRI 等 ${ }^{\left[{ }^{[00}\right]}$ 采用磁流变抛光技术改善 硅的表面光洁度, 并将单点金刚石切削和磁流变抛 光工艺对材料表面光洁度的影响进行了对比。结果 表明, MRF 能够有效地去除车削留下的痕迹。车削 后的沟槽在单点金刚石切削后的表面清晰可见, 但 在 MRF 后这些沟槽被有效去除, 如图 10 所示。较 小的间隙、较高的轮速和较大的电流有助于获得更 小的表面粗粘度， $R_{a}$ 最低可达 $0.607 \mathrm{~nm}$ 。

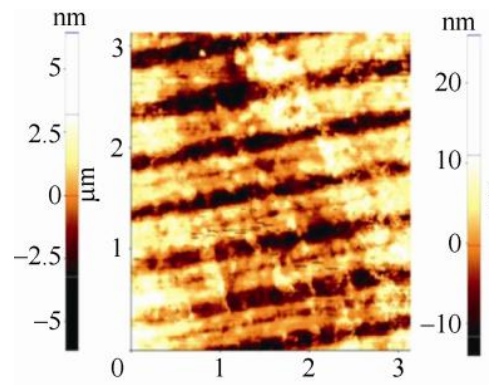

(a) 金刚石切削后的硅

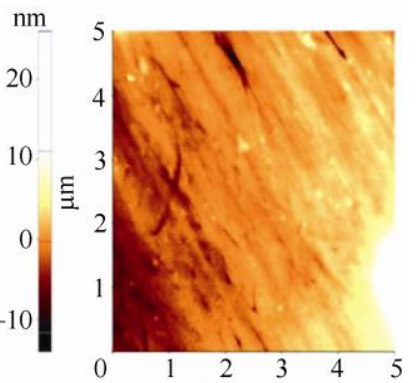

(b) 磁流变抛光后的硅
图 10 AFM 显微结构图 ${ }^{[40]}$

磁流变抛光过程中, 剪切应力是影响材料去除 效果的主要因素, 而压力的作用却往往会被忽略。 为了证明压力的重要性, LIU 等 ${ }^{[41]}$ 基于压力和剪切 应力的协同效应建立了 MRF 去除函数模型, 并结 合浸入深度、磁流变液黏度、抛光转速等参数, 验 证了该模型的精确性。结果表明, 与单一的剪切应 力诱导材料去除模型相比, 涉及压力的模型能够更 好地与去除函数保持一致。当压力指数取 $1 / 16$ 时, 模型在去除函数图形、峰值去除率、体积去除率等 方面与实际抛光点最匹配。该工作在补充磁流变抛
光机理研究方面欠缺的同时也为磁流变抛光的材料 去除行为研究提供了新的视角。磁流变抛光还可用 于测定材料亚表面损伤深度。SHAFRIR 等 ${ }^{[42]}$ 通过磁 流变抛光对微磨削后的 WC-Ni 复合材料表面形貌 进行了相关研究。结果表明, 采用磁流变抛光斑点 法可对亚表面损伤进行测试，揭示微磨削后材料亚 表面损伤的真实深度。适当的磁流变抛光确实能够去 除微磨削引起的表面变形层, 然而当去除过量时也会 导致择优抛光和材料中粘结剂去除的情况发生。

由于磁流变抛光具有表面粗䊁度小、加工面形精 度高、表面损伤小、易于实现计算机数控等优点, 近 年来一直是超精密加工领域的研究热点, 科研人员在 磁场效应的基础上又相继提出了集群磁流变抛光 ${ }^{[43]}$ 、 组合磁流变抛光 ${ }^{[44]}$ 、超声波磁流变复合抛光 ${ }^{[5]}$ 等优化 技术。然而, 目前磁流变抛光在技术实现上仍存在磁 流变液的研制和抛光过程中的柔性化控制等难题 ${ }^{[46]}$ 。

\section{4 力流变抛光}

力流变抛光(Force-induced rheological polishing, FRP) 是近年来新兴的一种超精密抛光技术。非牛顿 流体在剪切力作用下会产生新的结构, 材料黏度、 硬度和内部阻力会增大(可逆变化)。利用非牛顿流 体制备特定的抛光液, 抛光过程中, 工件与抛光液 之间进行相对运动, 非牛顿流体黏度急剧增大, 瞬 间呈现类似 “固体” 特性，增强对磨粒的把持力， 在加工位置形成一个柔性 “固着” 磨具, 从而实现 工件表面高效高质量抛光。

浙江工业大学吕冰海等 ${ }^{[47-49]}$ 率先提出了一种基 于非牛顿流体剪切增稠效应的新型抛光方法(Shear thickening polishing, STP), 并对其加工原理进行了 诠释, 如图 11 所示。非牛顿流体所受剪切应力大于 某一临界值时, 流体中高质量分数的固体胶态粒子 间的水合作用大于布朗作用力和分子键作用力, 促 使 “粒子簇” 的形成，这类 “粒子簇” 对流体的流 动起到很大的阻碍作用, 导致流体表观黏度急剧增 大，并呈现出宏观的固态特性。由于 “粒子簇” 的 形成是不稳定的, 随着剪切应力的减小, “粒子簇” 重新分解并与介质建立新的平衡, 因此, 流体的 “剪 切增稠” 现象是可逆的。图 12 为剪切增稠抛光的材 料去除过程图, 剪切增稠效应下, 流体中的固态粒 子迅速聚集并将磨粒把持包裹其中，形成柔性 “固 着” 磨具, 当剪切力 $F_{j}$ 大于磨粒所受阻力 $F_{z}$ 时, 工 件粗糙表面凸起的微峰被大部分去除; 当 $F_{j}<F_{z}$ 时, 形成的 “粒子簇” 又逐渐变化为松散状态, 流体恢 复至初始流动状态。除理论研究外, 吕冰海等 ${ }^{[49]}$ 还 对 $\Phi 20 \mathrm{~mm}$ 的 GCr15 轴承钢圆柱进行了加工试验, 
经过 $90 \mathrm{~min}$ 的 STP 后, 表面粗糙度 $R_{a}$ 由 $105.95 \mathrm{~nm}$ 降至 $5.99 \mathrm{~nm}$, 材料去除率达到 $2.1 \mu \mathrm{m} / \mathrm{h}$, 如图 13 所示, 证明 STP 确能实现工件的高效高质量抛光。

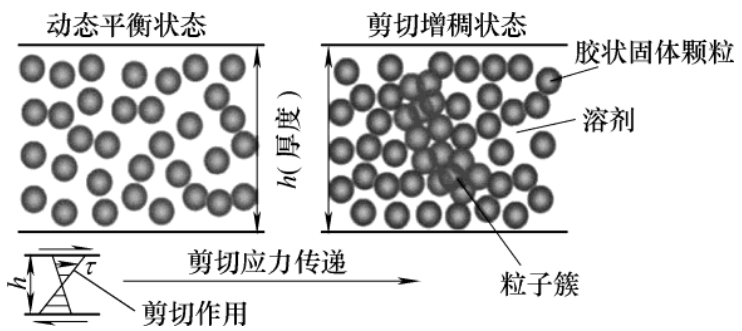

图 11 剪切增稠机理图 ${ }^{[48]}$

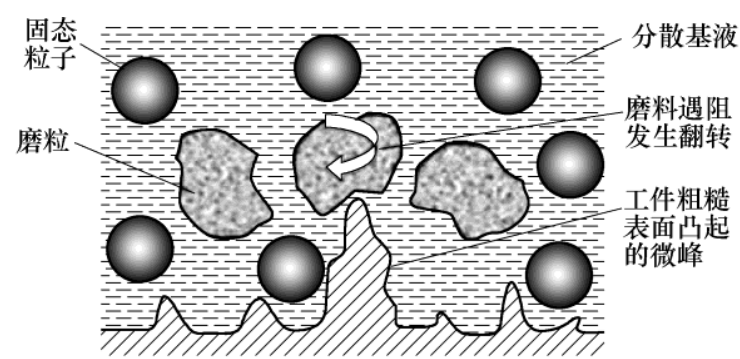

(a) 未发生剪切增稠

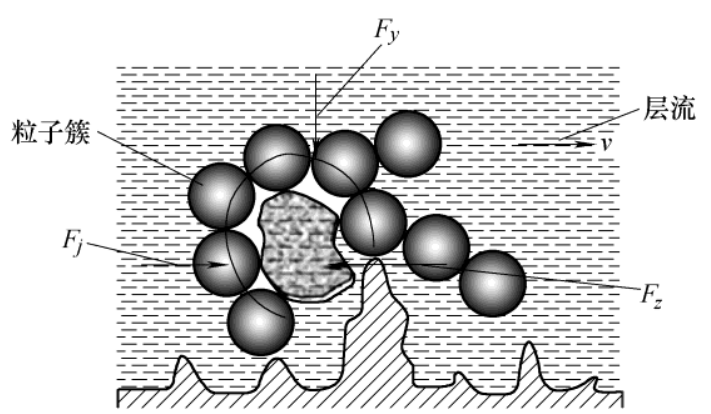

(b) 发生剪切增稠

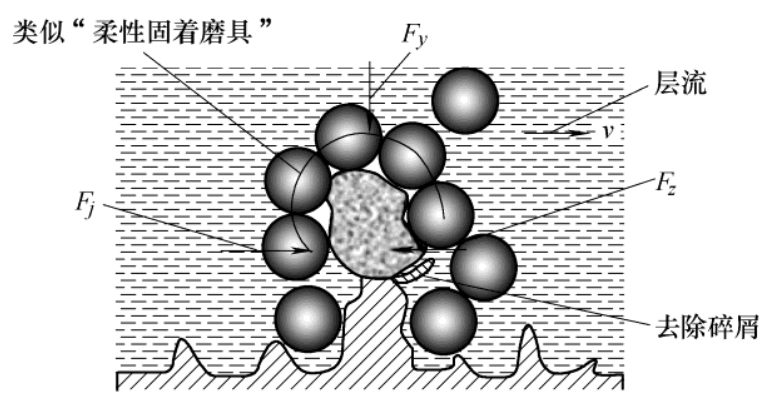

(c) 剪切增稠效应增强

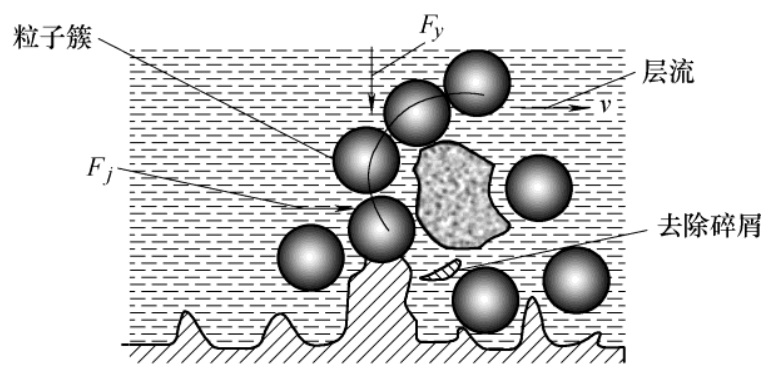

(d) 增稠效应减弱

图 $12 \mathrm{STP}$ 微观去除过程图 ${ }^{[49]}$

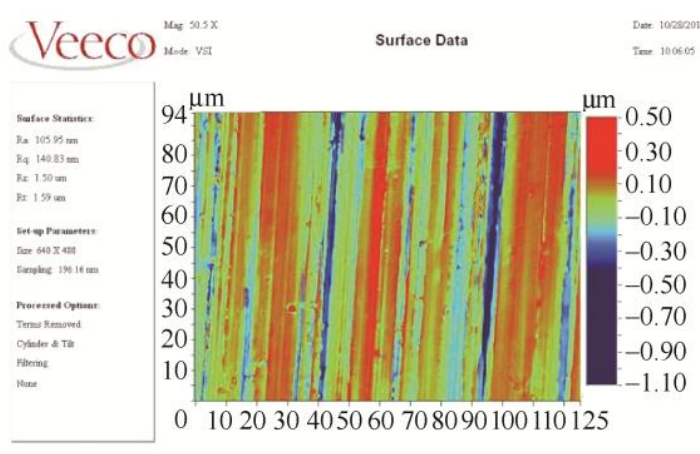

(a) 抛光前

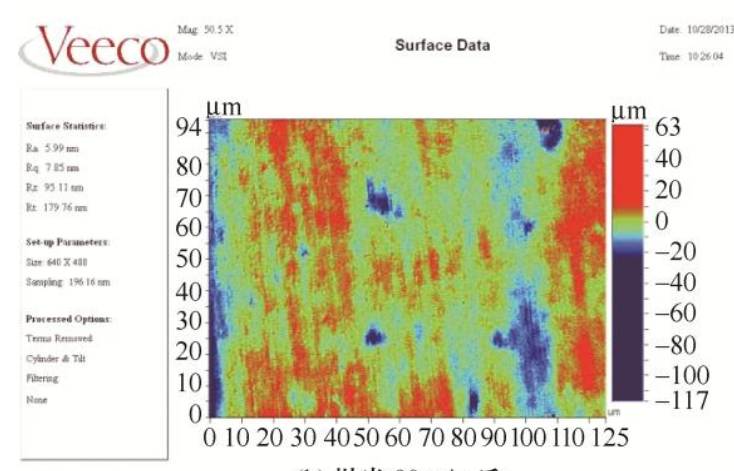

(b) 抛光 $90 \mathrm{~min}$ 后

图 13 剪切增稠抛光前后表面微观形貌 ${ }^{[49]}$

传统机械抛光常采用固定的研磨垫, 内嵌金刚 石等磨粒, 但埋置的颗粒深度和数量都随机的, 并伴 随有磨粒脱落的问题。日本九州大学土肥俊郎等 ${ }^{[50-51]}$ 从抛光垫和设备的角度考虑, 提出一种与 STP 原理 类似的新型抛光方法——剪切膨胀抛光(Dilatancy polishing)。利用粘弹性材料制备特定的抛光垫并 结合高压、高速条件下抛光垫的 “剪切膨胀” 效 应, 增强基体对磨粒的把持力的同时保证了工件表 面的受力均匀性, 使得工件与抛光垫均匀贴合从而 提高了抛光过程中材料的去除率, 减少局部应力集 中对工件表面的损伤, 实现了工件的高效、高精度 抛光。此外, 由于抛光垫的剪切膨胀特性, 加工过 程中可根据工艺需要对抛光压力和转速进行调整, 确保同一抛光垫低速、中速、高速多抛光步骤的使 用, 大大减少了时间和成本。结果表明, 在低-中 压力/速度条件下, 该方法的材料去除率比金属 $(\mathrm{Sn})$ 板抛光加工高出 3 倍以上, 能达到更低的表面粗粘 度, 且表面划痕及损伤更少。在 $1000 \mathrm{kPa}$ 的高抛 光压力下, 拋光垫对 $\mathrm{SiC}$ 材料的去除率是金属 $(\mathrm{Sn})$ 板的 10 倍。

由于力流变抛光是一种新兴的超精密抛光技 术, 现阶段主要针对的是其机理和工艺的研究, 暂 时还没有关于加工铇方面的研究报道。力流变抛光 作为一种新型柔性抛光技术，具有成本低、磨粒分 散性高、面性适应性好等优点, 其加工过程中不会 
因化学液的介入改变材料表层材质, 能够避免材料 表面化学产物的形成, 且不存在抛光液污染的问题。 该技术符合 PFM-W 的机械加工需求, 除此之外还 可广泛应用于各类材料的平面抛光甚至复杂曲面的 超精密抛光, 有望发展成为一种新的高效、高质量 抛光方法。

\section{PFM-W 超精密抛光技术比较}

超精密抛光是降低工件表面粗糙度、去除损伤 层, 获得高表面精度和表面质量的终加工手段。除 上述抛光方法之外, 目前新兴的抛光技术还有气囊 抛光、激光抛光、离子束抛光等。然而这些方法或 者抛光效率太低, 或者加工成本偏高, 或者抛光不 易控制, 各自存在一定的不足, 不适用于 PFM-W 表面高效高质量抛光。相较而言, CMP、ECP、MRF 及 FRP 具有较好的加工效率并能够实现材料高表面 质量抛光。

现阶段 PFM-W 的抛光问题主要有两点: (1) 高 效高质量抛光; (2) 化学产物污染, 即需要避免材料 表层在抛光过程中受化学液作用形成氧化铇, 从而 保证材料的抗氧化性和抗辐照性能, 延长反应堆使 用寿命。核聚变反应堆工况下, 氧化铇具有较强的 挥发性, 对反应堆第一壁产生极大的破坏, 这也是 为何目前科研人员试图在铇中掺杂 $\mathrm{Cr} 、 \mathrm{Si}$ 等合金元 素以期避免氧化铇的产生从而提高材料抗氧化性的 主要原因之一。

虽然化学机械抛光和电化学抛光均可实现 PFM-W 的高效高质量抛光和工件表面纳米级微 小单位的去除, 但其化学抛光液势必会腐蚀铇, 导致材料表面氧化铇的形成, 相当于改变了材料 的表层材质, 造成化学产物污染, 加剧 PFM-W 的氢/氦滞留和辐照损伤程度, 影响聚变堆的使用 寿命。此外, 上述两种抛光方法还存在化学液环 境污染的问题。

磁流变抛光和力流变抛光由于加工效率高、表 面损伤小、磨粒分散性高等优点, 是目前业内较为 认可的抛光新方法。两种抛光方法均可在实现 PFM-W 表面高效高质量抛光的前提下避免因化学 液作用改变 PFM-W 表层材质而形成氧化铇的问题, 同时还可避免化学液的环境污染。综上所述, 磁流 变抛光和力流变抛光是现阶段较为适合 PFM-W 表 面超精密加工的抛光方法, 有望实现 PFM-W 高效、 高质量抛光的同时提高材料的抗辐照性能。

\section{PFM-W 超精密抛光发展趋势}

综上所述, 虽然国内外学者长期以来在超精密 抛光方面进行了大量研究, 但也只倾向于抛光方法 原理研究和工艺优化, 针对 PFM-W 的抛光研究甚 少。而在核材料领域, 科研人员主要还是从材料学 角度研究 PFM-W 的抗辐照行为, 有关机械加工对 PFM-W 表面组织形貌和抗辐照性能的影响同样鲜 有报道。

随着国际上一批托卡马克装置成功建设和运 行, 磁约束聚变研究取得了一系列重大成果, 目前 已具备建造和运行反应堆级托卡马克试验装置科学 技术和工程建设条件 ${ }^{[52-53]}$ 。未来核聚变装置中, 铇 的使用量将超过 60 吨。实际运行工作前, 对 PFM-W 表面进行可规模化高效高质量加工处理从而提升其 抗辐照性能便显得尤为重要。因此, 有必要探索和 研究适用于 PFM-W 的高效、高质量、环保型的抛 光新方法, 并对抛后材料表面质量和抗辐照性能变 化进行深入研究, 进而推进核聚变工程化应用进程。 为了实现 PFM-W 的高效高精密加工, 建议应从以 下几个方面进行深入研究。

(1) 探索适合 PFM-W 的高效、高质量、环保型 的抛光新方法, 并借助现代材料分析测试手段, 从 表面畸变、亚表层损伤、表面粗䊁度、加工硬化层、 残余应力等角度考虑, 探究抛光对材料表面组织形 貌和表面质量的影响规律, 为优化新型抛光方法提 供理论基础;

（2）基于上述研究，探索表面形貌和表面质量 对 PFM-W 抗辐照行为的影响机理, 研究不同表面 形貌和表面质量铇的抗辐照性能差异, 揭示抛光对 PFM-W 抗辐照性能影响规律, 最终验证服役于辐照 环境下 PFM-W 机械加工的必要性;

(3) 结合计算仿真和加工试验, 建立材料去除 模型，系统分析抛光过程中不同抛光时间、抛光压 力、转速等工艺参数对材料去除率和表面粗鋉度的 影响, 并根据模型和试验结果优化抛光工艺, 建立 完善的 PFM-W 超精密加工工艺规程, 为未来 PFM-W 的超精密抛光奠定坚实的理论和技术基础。

此外, PFM-W 抛光新方法同样适用于除铇之外 其他硬脆材料的精密加工。

总之, 通过材料学和超精密抛光学科的交叉融 合, 未来必将促成 PFM-W 抛光技术以及核聚变工 程化的进一步发展。 


\section{参 考 文 献}

[1] KNASTER J, MOESLANG A, MUROGA T. Materials research for fusion[J], Nature Physics, 2016, 12: 424-434.

[2] WAN Y, LI J, LIU Y, et al. Overview of the present progress and activities on the CFETR[J]. Nuclear Fusion, 2017, 57: 102009.

[3] KULCINSKI G L, RADEL R F, Davis A. Near term, low cost, $14 \mathrm{MeV}$ fusion neutron irradiation facility for testing the viability of fusion structural materials[J]. Fusion Engineering and Design, 2016, 109-111: 1072-1076.

[4] PITTS R A, CARPENTIER S, ESCOURBIAC F, et al. Physics basis and design of the ITER plasma-facing components[J]. Journal of Nuclear Materials, 2011, 415(1): 957-964.

[5] RAFFRAY A R, NYGREN R, WHYTE D G, et al. High heat flux components-readiness to proceed from near term fusion systems to power plants[J]. Fusion Engineering and Design, 2010, 85(1): 93-108.

[6] BOLT H, BARABASH V, KRAUSS W, et al. Materials for plasma-facing components of fusion reactors[J]. Journal of Nuclear Materials, 2004, 329-333: 67-73.

[7] SHIMADA M, COSTLEY A.E, FEDERICI G, et al. Overview of goals and performance of ITER and strategy for plasma-wall interaction investigation[J]. Journal of Nuclear Materials， 2005， 337: 808-815.

[8] 吴玉程, 林锦山, 罗来马, 等. 面向等离子体铇基材料 的辐照损伤行为研究现状 [J]. 机械工程学报, 2017, 53(8): 25-34.

WU Yucheng, LIN Jinshan, LUO Laima, et al. Irradiation damage behavior research statues of tungsten-matrix materials facing plasma[J]. Journal of Mechanical Engineering, 2017, 53(8): 25-34.

[9] 周张健, 钟志宏, 沈卫平, 等. 聚变堆中面向等离子体 材料的研究进展[J]. 材料导报, 2005, 19(12): 5-12. ZHOU Zhangjian, ZHONG Zhihong, SHEN Weiping, et al. The development of plasma facing materials for fusion reactor[J]. Materials Reports, 2005, 19(12): 5-12.

[10] DAVIS J W, BARABASH V R, MAKHANKOV A, et al. Assessment of tungsten for use in the ITER plasma facing components[J]. Journal of Nuclear Materials, 1998, 258-263: 308-312.

[11] 孙兆轩, 李强, 王万景, 等. ITER 偏滤器铇/铜穿管模 块的高热负荷测试及事后分析 $[\mathrm{J}]$. 核聚变与等离子体 物理, 2017，37(4): 446-451.
SUN Zhaoxuan, LI Qiang, WANG Wanjing, et al. High heat flux test and post examination of ITER divertor $\mathrm{W} / \mathrm{Cu}$ monoblock mock-ups[J]. Nuclear Fusion and Plasma Physics, 2017, 37(4): 446-451.

[12] HIRAI T, PANAYOTIS S, BARABASH V, et al. Use of tungsten material for the ITER divertor[J]. Nuclear Materials and Energy, 2016, 9: 616-622.

[13] YOSHIDA N, IWAKIRI H, TOKUNAGA K, et al. Impact of low energy helium irradiation on plasma facing metals[J]. Journal of Nuclear Materials, 2005，337-339: 946-950.

[14] ALIMOV V.K, ROTH J. Hydrogen isotope retention in plasma-facing materials: Review of recent experimental results[J]. Physica Scripta, 2007, T128: 6-13.

[15] SHU W M, KAWASUSO A, YAMINISHI T. Recent findings on blistering and deuterium retention in tungsten exposed to high-fluence deuterium plasma[J]. Journal of Nuclear Materials, 2009， 386-388: 356-359.

[16] OGORODNIKOVA O V , KLIMOV K S , POSKAKALOV A G, et al. Deuterium and helium retention in W with and without He-induced W 'fuzz' exposed to pulsed high-temperature deuterium plasma[J]. Journal of Nuclear Materials， 2019， 515: 150-159.

[17] XU Q, SATO K, YOSHIIE T. Investigation of the interaction of He and D in FeBSi alloy[J]. Philosoophical Magazine Letters, 2013, 93: 560-565.

[18] CAO X Z, XU Q, SATO K, et al. Effects of dislocations on thermal helium desorption from nickel and iron[J]. Journal of Nuclear Materials, 2011，417: 1034-1037.

[19] SUGANO R, MORISHITA K, KIMURA A, et al. Microstructural evolution in $\mathrm{Fe}$ and $\mathrm{Fe}-\mathrm{Cr}$ model alloys after $\mathrm{He}^{+}$ion irradiations[J]. Journal of Nuclear Materials, 2004, 329-333: 942-946.

[20] CHEN H, LUO L, CHEN J, et al. Effects of zirconium element on the microstructure and deuterium retention of $\mathrm{W}-\mathrm{Zr} / \mathrm{Sc}_{2} \mathrm{O}_{3}$ composites[J]. Scientific Reports, 2016, 6: 32678 .

[21] 雷红, 雒建斌, 张朝辉. 化学机械抛光技术的研究进展 [J]. 上海大学学报, 2003, 9(6): 494-502.

LEI Hong, LUO Jianbin, ZHANG Chaohui. Advances in chemical mechanical polishing[J]. Journal of Shanghai University, 2003, 9(6): 494-502.

[22] 梅广益, 张克华, 文东辉. 抛光液化学成分对铇化学机 械抛光效果的影响研究[J]. 机械制造, 2010, 48(12): 88-91. 
MEI Guangyi, ZHANG Kehua, WEN Donghui. Study on influence of chemical composition of polishing fluid on polishing result for tungsten[J]. Machinery, 2010, 48(12): $88-91$.

[23] 贾英茜, 牛新环, 王现涁. 铇 CMP 中碱性抛光液组分 间化学作用及其影响 [J]. 微纳电子技术, 2015, 52(5): 334-338.

JIA Yingqian, NIU Xinhuan, WANG Xianbin. Chemical interaction and influence of alkaline slurry compositions in tungsten CMP $[\mathrm{J}]$. Micronanoelectronic Technology, 2015, 52(5): 334-338.

[24] SEO Y J, KIM N H, LEE W S. Chemical mechanical polishing and electrochemical characteristics of tungsten using mixed oxidizers with hydrogen peroxide and ferric nitrate[J]. Materials Letters, 2006, 60: 1192-1197.

[25] LIM G, LEE J H, KIM J, et al. Effects of oxidants on the removal of tungsten in CMP process[J]. Wear, 2004, 257: 863-868.

[26] JEONG Y A, PODDAR M K, RYU H Y, et al. Investigation of particle agglomeration with in-situ generation of oxygen bubble during the tungsten chemical mechanical polishing (CMP) process[J]. Microelectronic Engineering, 2019, 218: 111133.

[27] LARSEN-BASSE J, LIANG H. Probable role of abrasion in chemo-mechanical polishing of tungsten[J]. Wear, 1999, 233-235: 647-654.

[28] JACQUET P A. Electrolytic polishing of metallic surfaces[J]. Metal Finishing, 1949, 47(5): 48-54.

[29] DATTA M, VERCRUYSSE D. Transpassive dissolution of 420 stainless steel in concentrated acids under electropolishing conditions[J]. Journal of Electrochemical Society, 1990, 137(10): 3016-3023.

[30] DENG H, HUANG R, LIU K, et al. Abrasive-free polishing of tungsten alloy using electrochemical etching $[\mathrm{J}]$. Electrochemistry Communications, 2017, 82: 80-84.

[31] WANG F, ZHANG X, DENG H. A comprehensive study on electrochemical polishing of tungsten[J]. Applied Surface Science, 2019， 475: 587-597.

[32] 刘奉妍, 何代华, 刘平, 等. 柠檬酸钠对电解抛光金属 铇的影响 [J]. 功能材料, 2018, 49(11): 11163-11167. LIU Fengyan, HE Daihua, LIU Ping, et al. Effect of sodium citrate on electrolytic polishing of tungsten[J]. Journal of Functional Materials, 49(11): 11163-11167.

[33] 王雪, 李成山, 于泽铭, 等. $\mathrm{NiW}$ 合金基带电化学抛
光过程研究 [J]. 稀有金属材料与工程, 2012, 41(6): 1075-1079.

WANG Xue, LI Chengshan, YU Zeming, et al. Electropolishing process research of $\mathrm{NiW}$ alloy substrates[J]. Rare Metal Materials and Engineering, 2012, 41(6): 1075-1079.

[34] 杜炳志, 漆红兰. 电化学抛光技术新进展 [J], 表面技术, 2007, 36(2): 56-58.

DU Bingzhi, QI Honglan. Development of electrochemical polishing technology $[\mathrm{J}]$. Surface Technology, 2007, 36(2): 56-58.

[35] LIU D, ZHENG L, LUO L, et al. An overview of oxidation-resistant tungsten alloys for nuclear fusion[J]. Journal of Alloys and Compounds, 2018, 765: 299-312.

[36] 罗来马, 黄科, 备祥, 等. 合金化改性铇基材料的组织 和性能研究与发展 $[\mathrm{J}]$. 机械工程学报, 2018, 54(8): 117-128.

LUO Laima, HUANG Ke, ZAN Xiang, et al. Research and development of alloy modified tungsten-based materials[J]. Journal of Mechanical Engineering, 2018, 54(8): 117-128.

[37] KORDONSKI W I, JACOBS S D, GOLINI D, et al. Vertical wheel magnetorheological finishing machine for flat, convex, and concave surfaces[J]. Optical Fabrication and Testing, 1996, 7: 146-149.

[38] 尹韶辉, 王永强, 李叶鹏, 等. 蓝宝石基片的磁流变化 学抛光试验研究[J]. 机械工程学报, 2016, 52(5): 80-87. YIN Shaohui, WANG Yongqiang, LI Yepeng, et al. Experimental study on magnetorheological chemical polishing for sapphire substrate[J]. Journal of Mechanical Engineering, 2016, 52(5): 80-87.

[39] 彭小强, 戴一帆, 李圣怡. 磁流变抛光的材料去除数学 模型[J]. 机械工程学报, 2004, 40(4): 67-70.

PENG Xiaoqiang, DAI Yifan, LI Shengyi. Material removal model of magnetorheological finishing $[\mathrm{J}]$. Journal of Mechanical Engineering, 2004, 40(4): 67-70.

[40] KHATRI N, MISHRA V, SHARMA R, et al. Improving the surface finish of diamond turned silicon with magneto-rheological finishing[J]. Materials Today : Proceedings, 2017, 4: 10158-10162.

[41] LIU J, LI X, ZHANG Y, et al. Predicting the material removal rate (MRR) in surface magnetorheological finishing (MRF) based on the synergistic effect of pressure and shear stress[J]. Applied Surface Science, 2020, 504: 144492. 
[42] SHAFRIR S N, LAMBROPOULOS J C, JACOBS S D. A magnetorheological polishing-based approach for studying precision microground surfaces of tungsten carbides[J]. Precision Engineering, 2007，31: 83-93.

[43] 阎秋生, 汤爱军, 路家斌, 等. 集群磁流变效应微磨头 平面研抛加工参数研究 [J]. 金刚石与磨料磨具工程, 2008(5): 66-70.

YAN Qiusheng, TANG Aijun, LU Jiabin, et al. An experimental study of planarization technique with instantaneous tiny grinding wheel cluster based on magnetorheological effect[J]. Diamond \& Abrasives Engineering, 2008(5): 66-70.

[44] 李智. 组合磁流变抛光的关键技术研究 [D]. 长沙: 湖南 大学, 2016 .

LI Zhi. Research on the key technology of combined magnetorheological finshing[D]. Changsha : Hunan University, 2016.

[45] ZHANG F, WANG H, LUAN D, Research on material removal of ultrasonic-magnetorheological compound finishing $[\mathrm{J}]$. International Journal of Machining and Machinability of Materials, 2007, 2(1): 50.

[46] 胡扬轩, 邓朝晖, 万林林, 等. 用于蓝宝石材料加工的 新型超精密抛光技术及复合抛光技术研究进展. 材料 导报，2018，32(9): 1452-1458.

HU Yangxuan, DENG Zhaohui, WAN Linlin, et al. Research progress of the novel ultra-precision polishing techniques and composite polishing techniques for sapphire material processing[J]. Materials Review, 2018, 32(9): 1452-1458.

[47] LI M, LYU B, YUAN J, et al. Shear-thickening polishing method[J]. International Journal of Machine Tools \& Manufacture, 2015, 94: 88-99.

[48] LI M, LYU B, YUAN J, et al. Evolution and equivalent control law of surface roughness in shear-thickening polishing[J]. International Journal of Machine Tools \& Manufacture, 2016, 108: 113-126.

[49] 李敏, 吕冰海, 袁巨龙, 等. 剪切增稠抛光的材料去除 数学模型 $[J]$. 机械工程学报, 2016, 52(7): 142-151.

LI Min, LÜ Binghai, YUAN Julong, et al. Material removal mathematics model of shear thickening polishing[J]. Journal of Mechanical Engineering, 2016, 52(7): 142-151.

[50] DOI T K, SESHIMO K, YAMAZAKI T, et al. Smart polishing of hard-to machine materials with an innovative dilatancy pad under high-pressure, high-speed, immersed condition[J]. ECS Journal of Solid State Science and Technology, 2016, 5(10): 598-607.

[51] DOI T K, YAMAZAKI T, SESHIMO K, et al. Development of innovative "dilatancy pad" realizing super high efficiency and high-grade polishing of $\mathrm{SiC}$ wide band gap semiconductor substrates $[\mathrm{C}] / /$ International Conference on Planarization/ CMP Technology, Kobe, 2014, 168-173.

[52] 李建刚. 我国超导托卡马克的现状及发展 $[\mathrm{J}]$. 中国科 学院院刊, 2007, 22(5): 404-410.

LI Jiangang. Present status and development of superconducting Tokamak research in China[J]. Bulletin of Chinese Academy of Sciences, 2007, 22(5): 404-410.

[53] 李建刚. 托卡马克研究的现状及发展 [J]. 物理, 2016, 45(2): $88-97$.

LI Jiangang. The status and progress of Tokamak research[J]. Physics, 2016，45(2): 88-97.

作者简介: 陈泓谕, 男, 1991 年出生, 博士, 讲师。主要研究方向为核 聚变材料研究、超精密加工技术及装备。

E-mail: hychen@zjut.edu.cn

袁巨龙(通信作者), 男, 1962 年出生, 教授, 博士研究生导师。主要研 究方向为超精密加工技术及装备。

E-mail: jlyuan@zjut.edu.cn 